\title{
Comparative mitogenomics indicates respiratory competence in parasitic Viscum despite loss of complex I and extreme sequence divergence, and reveals horizontal gene transfer and remarkable variation in genome size
}

\author{
Elizabeth Skippington ${ }^{1,3}$, Todd J. Barkman², Danny W. Rice ${ }^{1}$ and Jeffrey D. Palmer ${ }^{1 *}$
}

\begin{abstract}
Background: Aerobically respiring eukaryotes usually contain four respiratory-chain complexes (complexes I-IV) and an ATP synthase (complex V). In several lineages of aerobic microbial eukaryotes, complex I has been lost, with an alternative, nuclear-encoded NADH dehydrogenase shown in certain cases to bypass complex I and oxidize NADH without proton translocation. The first loss of complex I in any multicellular eukaryote was recently reported in two studies; one sequenced the complete mitogenome of the hemiparasitic aerial mistletoe, Viscum scurruloideum, and the other sequenced the $V$. album mitogenome. The $V$. scurruloideum study reported no significant additional loss of mitochondrial genes or genetic function, but the $V$. album study postulated that mitochondrial genes encoding all ribosomal RNAs and proteins of all respiratory complexes are either absent or pseudogenes, thus raising questions as to whether the mitogenome and oxidative respiration are functional in this plant.

Results: To determine whether these opposing conclusions about the two Viscum mitogenomes reflect a greater degree of reductive/degenerative evolution in $V$. album or instead result from interpretative and analytical differences, we reannotated and reanalyzed the $V$. album mitogenome and compared it with the $V$. scurruloideum mitogenome. We find that the two genomes share a complete complement of mitochondrial rRNA genes and a typical complement of genes encoding respiratory complexes II-V. Most Viscum mitochondrial protein genes exhibit very high levels of divergence yet are evolving under purifying, albeit relaxed selection. We discover two cases of horizontal gene transfer in $V$. album and show that the two Viscum mitogenomes differ by 8.6-fold in size (66 kb in V. scurruloideum; $565 \mathrm{~kb}$ in V. album).

Conclusions: Viscum mitogenomes are extraordinary compared to other plant mitogenomes in terms of their wide size range, high rates of synonymous substitutions, degree of relaxed selection, and unprecedented loss of respiratory complex I. However, contrary to the initial conclusions regarding V. album, both Viscum mitogenomes possess conventional sets of rRNA and, excepting complex I, respiratory genes. Both plants should therefore be able to carry out aerobic respiration. Moreover, with respect to size, the $V$. scurruloideum mitogenome has experienced a greater level of reductive evolution.
\end{abstract}

Keywords: Mitogenome, Mutation rate, Complex I, Mistletoes, Parasitic plants, Respiratory competence

\footnotetext{
* Correspondence: jpalmer@indiana.edu

${ }^{1}$ Department of Biology, Indiana University, Bloomington, IN 47405, USA

Full list of author information is available at the end of the article
} 


\section{Background}

Almost all eukaryotes capable of carrying out aerobic respiration contain four multi-subunit electron-transfer complexes (complexes I-IV) that are more-or-less embedded in the inner mitochondrial membrane. Three of these complexes (I, III, and IV) translocate protons across this membrane to generate a proton gradient used by the mitochondrial ATP synthase (complex V) to phosphorylate ADP to ATP. In all respiring eukaryotes, the mitogenome contains genes for subunits of at least one of these complexes. Most often, as in all animals and virtually all plants, genes for subunits of complexes I, III, IV, and V are present in mitochondrial DNA. In several lineages of aerobic eukaryotes, complex I has been lost, with an alternative, nuclear-encoded NADH dehydrogenase known or thought to bypass complex I and oxidize $\mathrm{NADH}$ without proton translocation, while the apicomplexan relative Chromera velia was recently shown to have uniquely lost (for an aerobe) both complexes I and III [1].

In June, 2015, we reported the complete sequence of the mitogenome of the aerial hemiparasite ("mistletoe") Viscum scurruloideum [2]. Most notable among several unusual features of this genome is its lack of the nine genes for complex I that are present in all other $300+$ examined angiosperms [3, 4], whereas it contains a typical complement of plant mitochondrial genes for complexes II-V and for ribosomal RNA. Because functional transfer to the nucleus of all nine missing complex I (nad) genes is prohibitively unlikely [2], we concluded that $V$. scurruloideum represents the first reported case of loss of complex I in any multicellular eukaryote.

In December 2015, Petersen et al. [5] reported a partial complement of mitochondrial genes for $V$. minimum and $V$. crassulae and a complete mitogenome sequence for $V$. album, known as the common or European mistletoe. On two key issues, this study reached opposite conclusions than those published for $V$. scurruloideum. These conclusions were 1) that "mitochondrial genes encoding proteins of all [Viscum] respiratory complexes are lacking or pseudogenized" and 2) that the mitochondrial rRNA genes are either missing or so divergent in Viscum that their mitochondria "would be unable to perform protein biosynthesis unless all the missing rRNA[s] ... are imported."

These opposing conclusions, which were not discussed in the V. album paper [5], as it did not cite the V. scurruloideum study, could largely or entirely reflect a greater degree of reductive/degenerative evolution of the mitochondrial gene repertoire in $V$. album relative to $V$. scurruloideum. Alternatively, they could result primarily from interpretational and analytical differences between the two Viscum studies $[2,5]$. Therefore, to address these issues, we have reannotated and reanalyzed the mitogenome of $V$. album using sequence-analysis parameter settings better suited for the high levels of sequence divergence in Viscum mitogenomes $[2,5-7]$ and compared the $V$. album genome to the $V$. scurruloideum genome. We show that the major conclusions reached in the $V$. album study [5] are incorrect: The two Viscum mitogenomes have a virtually identical complement of vertically inherited protein and rRNA genes, including a full set of rRNA genes and an essentially full set of complex II-V genes, with the $V$. album mitogenome containing 21 intact protein genes rather than the 12 reported by the $V$. album study [5]. We also show that the anomalously low level of divergence exhibited by two $V$. album genes is most likely the result of their recent acquisition via horizontal gene transfer (HGT), rather than evidence that they "may [be]...the only truly functional genes" in the $V$. album mitogenome. Finally, we report that the $V$. album and $V$. scurruloideum mitogenomes exhibit a remarkable 8.6-fold difference in size.

\section{Results and Discussion}

Reassessing mitochondrial protein gene loss in Viscum album

To identify protein and rRNA genes in the $V$. album mitogenome, Petersen et al. [5] compared it against local databases of mitochondrial protein and rRNA genes from 20 unnamed plant mitogenomes using BLASTX and BLASTN [8], respectively, with unspecified BLASTparameter settings. Absence of BLAST high-scoring segment pairs (HSPs) was considered evidence for gene loss. This approach is problematic because extensive sequence divergence, as reported in both $V$. album and $V$. scurruloideum [2, 5], can obscure the underlying sequence similarity possessed by homologous genes [9]. The absence of HSPs can be indicative of gene loss, but at high levels of sequence divergence, and dependent on various parameter settings, BLAST searches frequently yield false negatives in homolog detection [10].

We therefore reannotated the $V$. album mitogenome using two complementary strategies. First, the $V$. album genome was compared to mitochondrial gene sequences from 33 complete angiosperm mitogenomes (listed in Additional file 1: Figure S1) using BLASTN 2.2.28+ [8] with the following parameter settings: word size $=7$, reward $=5$, penalty $=-4$, gapopen $=8$, and gapextend $=6$. These sensitive settings allowed us to detect divergent gene sequences that went undetected in the published $V$. album annotation [5]. Second, the rRNA- and proteingene sequences of the $V$. scurruloideum mitogenome [2] were compared to the $V$. album genome using BLASTN with default parameter settings. This provided additional power to delineate $V$. album mitochondrial gene and exon boundaries, as these genes are extremely divergent relative to genes of all examined non-Viscum angiosperms, but highly similar to those of $V$. scurruloideum (Fig. 1). Both strategies were needed because, although using the $V$. scurruloideum genes as BLAST queries is in general more 


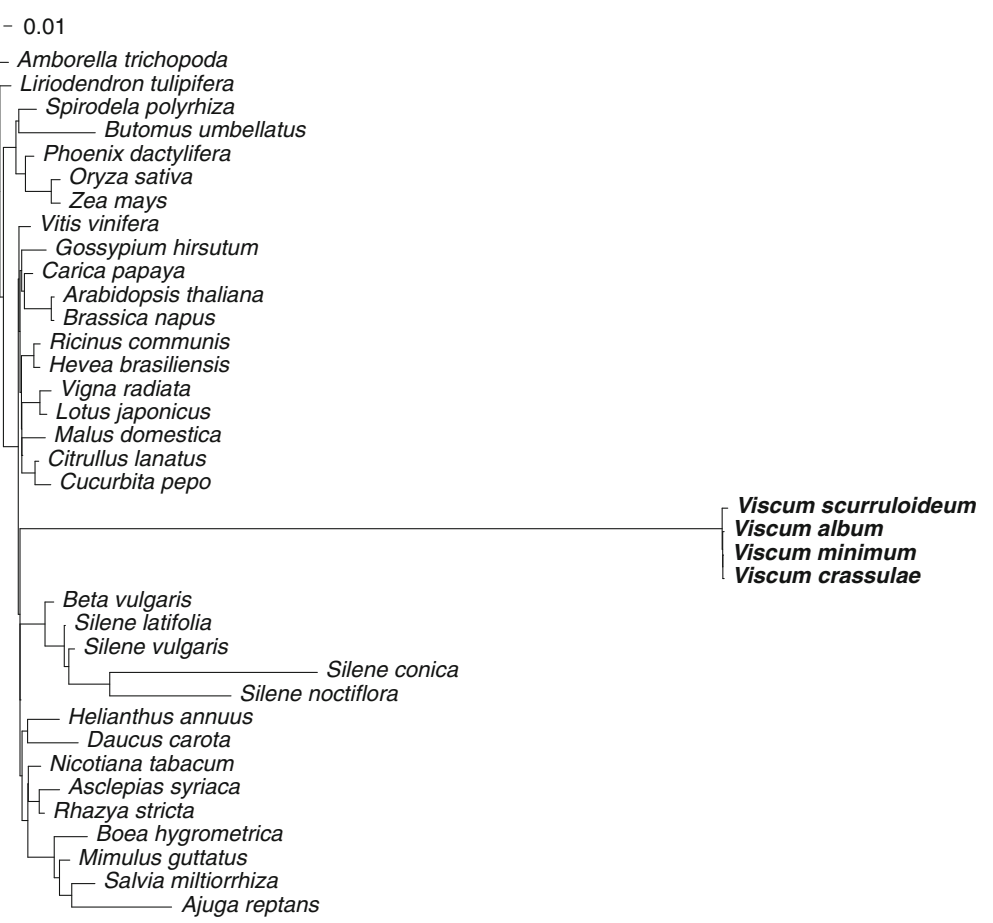

Fig. 1 Viscum genes are extremely divergent relative to genes of other angiosperms with sequenced mitogenomes (see Fig. 3 for comparison to two divergent angiosperms, Plantago and Pelargonium, for which only a few gene sequences are available). A constrained topology was enforced for maximum likelihood (RAXML, GTRGAMMA) branch-length estimation on the basis of a concatenated alignment of all three codon positions of the nine best-conserved protein genes in Viscum scurruloideum (atp 1, atp6, atp9, ccmC, cob, cox1, cox2, cox3, rps 12; see [2])

effective, using this approach alone would have missed any genes present in $V$. album, but absent from $V$. scurruloideum.

Angiosperm mitogenomes almost invariably share a set of 24 "core" protein genes, but can differ substantially in their inventories of a further 17 variably-present protein genes $[3,4,11]$. Contrary to Petersen et al. [5], but as expected based on the results of Skippington et al. [2] and other comparative studies among angiosperms [3, 11], BLAST searches yielded HSPs between the $V$. album mitogenome and most of the 24 core protein genes (Fig. 2). Using these HSPs as a starting point, we were able to annotate 15 of the 24 core protein genes as intact in $V$. album, 13 of which are likely to be functional (as described in the HGT section below, the $V$. album ccmB and matR genes were probably acquired recently via HGT and are unlikely to be functional). These include genes of complexes III (cob), IV (cox1, cox 2, and cox3) and V (atp1, atp4, atp6, atp8, and atp9), as well as cytochrome c biogenesis proteins $(c c m B, c c m C, c c m F c$, and $c c m F n)$, maturase $m a t R$, and protein transporter $m t t B$. Notably, $a t p 4, a t p 8, c c m F c$, and $m t t B$ were missed in the original annotation [5], presumably due to their extensive sequence divergence $(41.5 \%, 56.0 \%, 51.1 \%$, and $63.2 \%$ nucleotide identity, respectively, with their counterparts in the Liriodendron mitogenome; Table 1). The only departure in the $V$. album mitogenome with respect to core genes is the apparent loss or pseudogenization of all nine nad genes (nad1, nad2, nad3, nad4, nad4L, nad5, nad6, nad7, and nad9). Although this is not at all unexpected in light of the loss of respiratory complex I in $V$. scurruloideum (for comprehensive discussion of this loss, see Skippington et al. [2]), it nonetheless provides useful confirmation. In particular, the fact that the mitogenome of $V$. album contains clearly pseudogenized remnants of most nad genes, whereas that of $V$. scurruloideum contains no such traces, further rules out the already remote possibility that the latter species somehow contains functional but essentially undetectable forms of these genes. Because our annotation of the V. album mitogenome conflicts with the original conclusion [5] that "mitochondrial genes encoding proteins of all respiratory complexes are lacking or pseudogenized," we will discuss evidence for the functionality of complexes II-V in a separate section.

Petersen et al. [5] identified only one (rps12) of the 17 genes of highly variable presence in angiosperms. Our analysis also found $r p s 12$, as well as five genes ( $r p l 16, r p s 3$, rps4, rps10, and sdh3) that were classified as missing [5]. In $V$. scurruloideum, we originally annotated $s d h 3$ as a pseudogene due to its truncated length (186 nt compared to $282 \mathrm{nt}$ in Liriodendron) [2], but in light of high sequence identity (97.3\%) with its 222 nt counterpart 


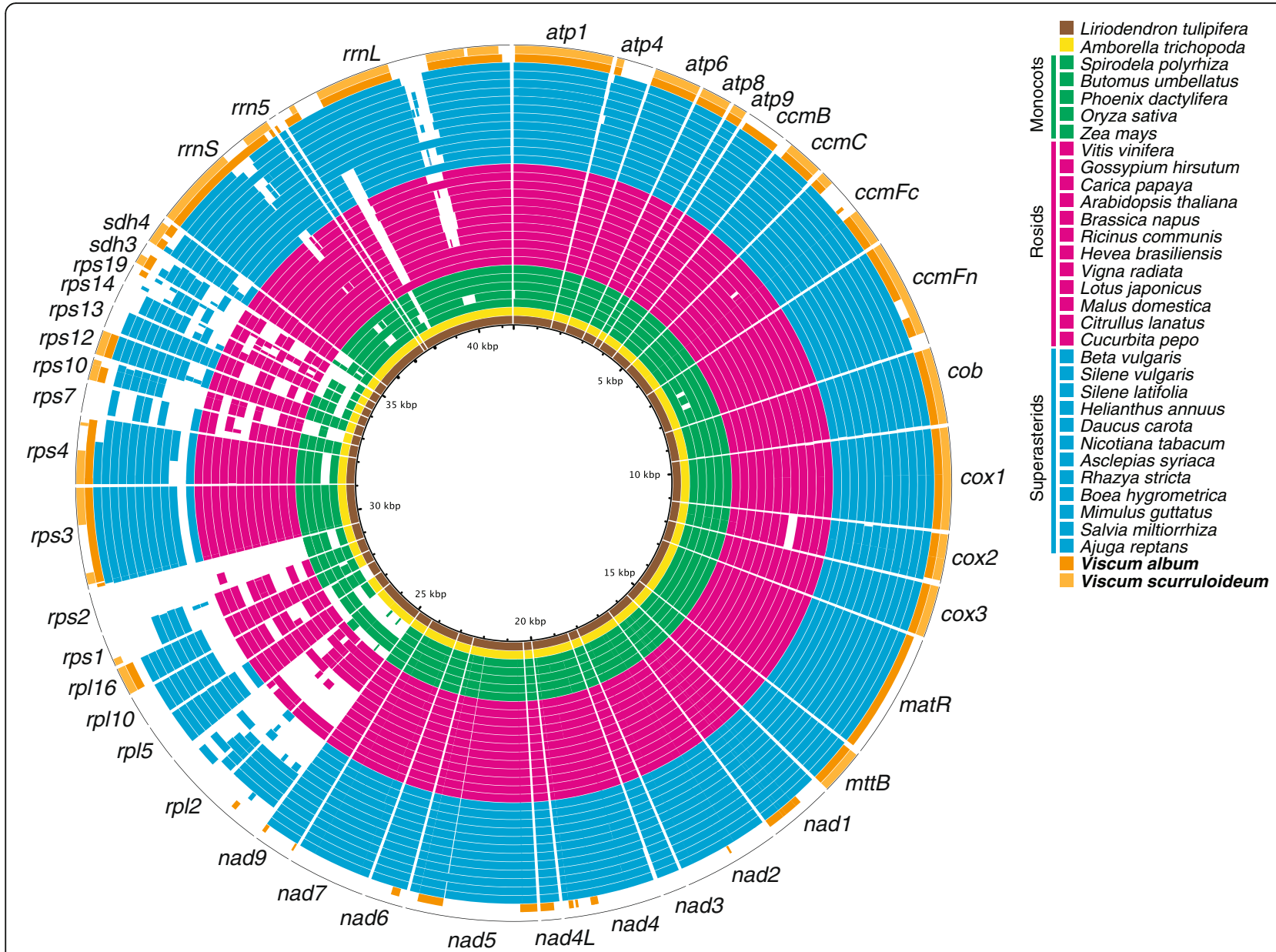

Fig. 2 Evidence for virtually identical content of intact protein and rRNA genes in V. album and $V$. scurruloideum. Shown is a genome-wide BLAST comparison of angiosperm mitochondrial rRNA- and protein-coding sequences. Rings show high-scoring segment pairs (HSPs) yielded by BLASTn searches of the corresponding query mitochondrial genome against Liriodendron rRNA- and protein-coding sequences (total length $=41,475 \mathrm{bp}$ ) with the following parameter settings: evalue $=0.1$, word_size $=7$, reward $=5$, penalty $=-4$, gapopen $=8$ gapextend $=6$, and perc_identity $=20$. The outer ring (of thin black segments) displays the Liriodendron sequences shown to scale. Inner rings correspond to completely sequenced angiosperm mitochondrial genomes of magnolids (brown), monocots (green), rosids (pink), Santalales (orange), and superasterids excluding Santalales (blue). For the Amborella ring, only HSPs involving native Amborella genes are shown. This figure was produced using BRIG [43]

in $V$. album, we have reannotated $s d h 3$ as putatively functional in both Viscum species. Altogether, we identified 21 putatively functional protein genes in the $V$. album mitogenome assembly (Additional file 1: Table S1 and Figure S1), compared to the 12 originally reported [5].

\section{A complete set of functional rRNAs in V. album}

Petersen et al. [5] state that "we were unable to locate 5S rRNA in any of the three Viscum species, and the recovered 26S rRNA-like sequences are so divergent that the genes are unlikely to be functional" and that "The identified sequences of the 18S rRNA genes are also very different from those of all other seed plants suggesting that they too are not functional." However, there was no evidence provided to support these claims regarding the $18 \mathrm{~S}$ and 26
rRNAs, including any quantitative sense - in terms of either phylogenetic or comparative sequence analysis of the level of divergence exhibited by the $V$. album rRNA sequences. We readily identified intact and almost certainly functional genes in $V$. album for the large subunit (LSU), small subunit (SSU) and $5 \mathrm{~S}$ rRNAs ( $r n \mathrm{~L}, r r n S$, $r r n 5$, respectively) (Fig. 2). It is true that all three of these genes are divergent in the context of angiosperm evolution (Additional file 1: Figure S2), with $r r n \mathrm{~L}, r r n \mathrm{~S}$, and $r r n 5$ sharing only $63 \%, 70 \%$, and $53 \%$ identity, respectively, with their counterparts in the relatively ancestral-like and slowly evolving Liriodendron mitogenome. However, there is a remarkable level of variation in the size, sequence, and structure of mitochondrial rRNAs across eukaryotes, a level that far exceeds the variation seen in rRNAs across prokaryotes, nuclear genomes, and plastid genomes, and a 
Table 1 Protein-gene divergence in Viscum album (Va) vs. Liriodendron tulipifera (Lir) and Va vs. V. scurruloideum (Vs)

\begin{tabular}{|c|c|c|c|c|c|c|c|c|}
\hline \multirow[t]{2}{*}{ Gene } & \multicolumn{3}{|c|}{ Coding sequence length (nt) } & \multicolumn{2}{|c|}{$\%$ nucleotide identity $^{a}$} & \multicolumn{3}{|c|}{ Va- Vs syn. and nonsyn. ${ }^{b}$} \\
\hline & Lir & Va & Vs & Va vs. Lir & Va vs. Vs & $d_{N}$ & $d_{S}$ & $d_{N} / d_{S}$ \\
\hline atp9 & 225 & 225 & 219 & 82.7 & 91.8 & 0.044 & 0.216 & 0.20 \\
\hline $\operatorname{rps} 12$ & 375 & 417 & 411 & 68.0 & 98.3 & 0.010 & 0.048 & 0.21 \\
\hline atp1 & 1527 & 1536 & 1536 & 73.1 & 99.8 & 0.001 & 0.004 & 0.22 \\
\hline $\operatorname{cox} 1$ & 1572 & 1602 & 1602 & 81.4 & 99.6 & 0.003 & 0.010 & 0.24 \\
\hline $\operatorname{cox} 3$ & 795 & 738 & 795 & 75.1 & 99.2 & 0.006 & 0.017 & 0.33 \\
\hline rps3 & 1554 & 1455 & 1518 & 50.4 & 97.6 & 0.018 & 0.044 & 0.41 \\
\hline $\mathrm{ccmFC}$ & 1326 & 1305 & 1218 & 51.1 & 96.3 & 0.029 & 0.067 & 0.43 \\
\hline sdh3 & 282 & 222 & 186 & 54.8 & 97.3 & 0.022 & 0.047 & 0.47 \\
\hline atp6 & 720 & 915 & 915 & 75.5 & 99.0 & 0.008 & 0.016 & 0.51 \\
\hline $\mathrm{ccmFn}$ & 1683 & 1776 & 1467 & 51.8 & 96.7 & 0.029 & 0.051 & 0.56 \\
\hline$m t t B$ & 720 & 765 & 789 & 63.2 & 96.9 & 0.029 & 0.042 & 0.68 \\
\hline atp4 & 558 & 471 & 474 & 41.5 & 98.5 & 0.014 & 0.020 & 0.69 \\
\hline$c o b$ & 1179 & 1209 & 1221 & 74.4 & 99.3 & 0.006 & 0.008 & 0.73 \\
\hline rps4 & 1035 & 1005 & 1224 & 51.8 & 96.1 & 0.037 & 0.050 & 0.75 \\
\hline rp/16 & 432 & 378 & 378 & 63.2 & 98.4 & 0.015 & 0.019 & 0.79 \\
\hline $\mathrm{ccmC}$ & 699 & 654 & 654 & 65.9 & 99.5 & 0.004 & 0.005 & 0.84 \\
\hline atp8 & 474 & 492 & 483 & 56.0 & 98.3 & 0.016 & 0.019 & 0.85 \\
\hline $\cos 2$ & 762 & 750 & 750 & 76.3 & 99.6 & 0.005 & 0.000 & n.a. \\
\hline rps10 & 357 & 273 & 273 & 59.4 & 99.6 & 0.006 & 0.000 & n.a. \\
\hline All genes ${ }^{c}$ & 16275 & 16188 & 16113 & 64.4 & 98.2 & 0.015 & 0.029 & 0.50 \\
\hline
\end{tabular}

adentity calculations exclude gap positions

${ }^{b}$ Synonymous $\left(d_{S}\right)$ and nonsynonymous $\left(d_{N}\right)$ site divergence

'Values are from a concatenate of all 19 protein genes

level against which the Viscum divergence pales. For example, the SSU and LSU rRNAs of animal mitochondria vary in size by a factor of 4.0 (513-2,036 nt) and 6.6 (529$3,487 \mathrm{nt}$ ), respectively [12]. A broad diversity of mitochondrial genomes do not even have complete SSU and LSU rRNA genes, with the gene fragments sometimes extensively scrambled in the genome, and with as many as 27 fragments for both genes combined in Plasmodium falciparum (e.g., $[13,14])$. Despite this divergence, those regions of the mitochondrial SSU and LSU rRNAs that assemble to form the catalytic core of each ribosomal subunit are conserved in all known mitochondria $[14,15]$.

Furthermore, we superimposed the $V$. album SSU and LSU rRNA sequences onto the secondary structures of mitochondrial SSU and LSU rRNAs from the angiosperms Oenothera berteriana and Zea mays, respectively (Additional file 1: SI Appendix, Figures S3 and S4, respectively). This shows that the $V$. album rRNAs share extensive sequence identity with the highly conserved regions of large and small subunit rRNAs and strongly implies the existence of rigid functional and structural constraints. We previously carried out similar analyses for $V$. scurruloideum that supported the functionality of its rRNAs [2]. Very few of the mutations in the $V$. scurruloideum rRNAs correspond to base-pairing nucleotides of the secondary structures, and most have been accompanied by compensatory base-pair changes (see Additional file 1: Figure S4 and S5 of [2]). The 18S rRNA of $V$. scurruloideum has 10 compensatory basepair changes relative to reference Oenothera berteriana, while the 23S rRNA has 23 compensatory changes relative to Zea mays. The high level of sequence conservation between the $V$. album and $V$. scurruloideum SSU and LSU rRNAs (95\% and 91\% identity, respectively, gaps excluded) is also consistent with their being functional.

A full 5S rRNA alignment is shown in Additional file 1: Figure S5. The small size and/or complex secondary structures of mitochondrial 5S rRNA make identification notoriously difficult [16]. Indeed, we originally annotated only a 38 nt-long sequence as $5 \mathrm{~S}$ rRNA in $V$. scurruloidum [2] because clear gene boundaries were difficult to ascertain. Pairwise alignment of this short sequence to the Liriodendron 5S rRNA yielded $87 \%$ identity, excluding gaps [2]. Here, using secondary structure analysis (Additional file 1: Figure S6) and sequence conservation (100\% identity, in fact) between the two Viscum sequences, we were able to extend the annotated gene length to $118 \mathrm{nt}$ 
in both species (as compared to 112-126 nt in most angiosperms). A recent study that used a mitochondrion-specific covariance model to screen mitochondrial genome sequences for $5 \mathrm{~S}$ rRNA identified more than 50 previously unrecognized homologs in mitochondrial genomes of various stramenopiles, red algae, cryptomonads, malawimonads and apusozoans and also showed that 5S rRNAs have a much larger structural variability than previously thought [16]. However, the divergence of the 5S rRNAs discovered by Valach et al. [16] far exceeds the divergence of the Viscum $5 \mathrm{~S}$ rRNAs relative to other angiosperms.

Six sequences were identified in the $V$. album mitogenome that could be folded into a typical tRNA structure (Additional file 1: Figure S7). These sequences correspond to two different copies each of $\operatorname{trn} W$ (cca) and $\operatorname{trnM}$ (cau), and one copy each of $\operatorname{trnK}(u u u)$, and $\operatorname{trn} G(g g c)$. Although the $V$. album mitogenome contains far fewer types of tRNA genes than most other angiosperms, even greater reduction in tRNA gene content (two, three, and three, respectively) has been reported for the $S$. conica, S. noctiflora, and $V$. scurruloideum mitogenomes [2, 17]. Moreover, several lineages of non-plant eukaryotes, including Trypanosoma brucei, Leishmania tarentolae, and Plasmodium falciparum, have no tRNA genes left in their mitogenome and are thus entirely dependent on import of nuclear-encoded tRNAs [18-20].

Petersen et al. [5] state that "Viscum mitochondria would be unable to perform protein biosynthesis unless all the missing rRNA, ribosomal proteins and tRNAs are imported". However, we are unaware of any precedent for the mitochondrial import of SSU and LSU rRNAs, nor, as described above, is there are reason to posit such import in Viscum. In contrast, many diverse eukaryotes import most or all of their mitoribosomal proteins and/ or tRNAs [21]. Angiosperm mitoribosomes contain at least 63 proteins, 48 of which were encoded by the nucleus of the ancestral angiosperm, with the genes for another 15 proteins having been functionally relocated to the nucleus numerous times during angiosperm evolution [3]. Indeed many lineages of angiosperms have evolved smaller sets of mitoribosomal protein genes than the five present in $V$. album and V. scurruloideum (Additional file 1: Figure S1; $[3,17])$. In conclusion, we see no reason to think that $V$. album mitochondria are either incapable of carrying out protein synthesis or require the unprecedented import of translational components.

\section{Reevaluating respiratory competence in the face of exceptional sequence divergence: evidence for a full set of functional complex II-V genes in V. album}

Phylogenetic analysis (Additional file 1: Figure S2) of the 21 protein genes present in V. album shows that, except for matR and $c c m B$ (see next section), all of these genes exhibit exceptionally high levels of divergence relative to most other angiosperms, including the two other sampled Santalales, Comandra umbellata (Comandraceae) and Amyema beccarri (Loranthaceae). Almost all of this divergence is located on the branch leading to the last common ancestor of Viscum (Table 1, Fig. 1, and Additional file 1: Figure S2). Our previous analyses of $V$. scurruloideum indicated that this divergence is largely a consequence of a highly elevated rate of synonymous substitutions [2]. Given the high levels of divergence observed in $V$. scurruloideum, we raised - and answered the question of whether the most divergent $V$. scurruloideum genes are potentially functional [2]. Because the highly divergent protein genes shared by $V$. album and $V$. scurruloideum are extremely similar (12 of 19 are $>98 \%$ identical in nucleotide sequence, and all but one are $>96 \%$ identical; see Table 1), and because Petersen et al. [5] inferred that all five respiratory and ATP-synthesis complexes are defunct in $V$. album, it is prudent to revisit and extend our arguments for the functionality of Viscum protein genes.

As in $V$. scurruloideum, the 19 divergent protein genes in $V$. album are, for the most part, encoded by ORFs of moderate to considerable length (Table 1). Thirteen are of equal or greater length than their counterparts in V. scurruloideum (Table 1). Considering the highly accelerated evolution of Viscum mitochondrial sequences, we previously argued that premature stop codons should have been introduced into most if not all V. scurruloideum genes that were not functional [2]. Indeed we showed that the probabilities of such ORFs being maintained by chance alone are extremely low [2]. By extension, therefore it is likely that the $V$. album ORFs are, by and large, functional, because they are all comparable in length to those found in $V$. scurruloideum (Table 1).

To further assess the level of divergence and functionality of Viscum protein genes, we estimated $d_{S}$ and $d_{N}$ values (Table 1, Additional file 1: Figure S8). Wherever possible, we included $V$. scurruloideum, $V$. minimum, and $V$. crassulae homologs in $d_{S}$ and $d_{N}$ trees. The patterns of divergence in the $d_{\mathrm{S}}$ trees (Additional file 1: Figure S8) are similar to those in the trees based on all three codon positions (Additional file 1: Figure S2). For almost all genes, the longest $d_{\mathrm{S}}$ branch is the one leading to the last common ancestor of Viscum, and the longest rootto-tip branches are those leading to the sampled Viscum species (Additional file 1: Figure S8). As previously concluded [2], this divergence is consistent with a highly elevated rate of synonymous substitutions operating across the mitogenome in Viscum. There is comparatively little divergence among the four sampled Viscum species, indicating that most nucleotide substitutions occurred in the Viscum lineage before these species diverged (Additional file 1: Figure S8). 
The $d_{N}$ values on the branch leading to Viscum are also highly elevated relative to other angiosperms (Additional file 1: Figure S8). Moreover, in a number of gene trees (most notably, those for atp1, atp6, $\mathrm{ccmC}$, $\operatorname{cob}, \operatorname{cox} 1, \operatorname{cox} 2$, and $\operatorname{cox} 3) d_{N} / d_{S}$ values are markedly higher on the ancestral Viscum branch compared to other angiosperm branches with high $d_{S}$ values (e.g., those leading to Silene noctiflora and S. conica). Furthermore, excluding cox2 and rps10, which have no synonymous-site differences between $V$. album and $V$. scurruloideum, the average pairwise $d_{N} / d_{S}$ value for $V$. album $/ V$. scurruloideum is 0.52 (Table 1), which is also unusually high. Importantly, however, in all cases $d_{N} / d_{S}$ for $V$. album $/ V$. scurruloideum is below 1.0, ranging from 0.20 to 0.85 (Table 1), which again suggests that most if not of all these protein genes are functional. These findings, together with those previously reported [2], suggest that many protein genes in Viscum are evolving under relaxed selection relative to most plants. To formally test for changes in selective strength, we used RELAX [22], a codon-based, branch-site random-effects method, to test the nine best conserved [2] Viscum protein genes (excluding matR and $c c m B$, see next section) for changes in selective strength. We find that selection is significantly relaxed along the Viscum branches in eight of the nine gene sets (selection intensity $k=0.17-0.70$; Table S2).
Finally, because it was implied [5] that the level of sequence divergence exhibited by $V$. album respiratory and ATP-synthesis genes is incompatible with them being functional, we sought to provide a broader phylogenetic context for this divergence. We therefore constructed protein trees for complex III protein Cob (cytochrome b) and complex IV protein Cox1 (cytochrome oxidase subunit 1), two of the best conserved proteins involved in respiration and ATP synthesis, using sequences chosen to reflect the breadth of Cob and Cox1 sequence diversity in eukaryotes. Although these two proteins are unusually divergent in Viscum relative to other flowering plants (Figs. 2 and 3), this divergence pales in comparison to their divergence in many other eukaryotes, including several groups within animals alone (Fig. 3). For example, Cob and Cox1 from the myxozoan Kudoa hexapunctata, which harbors the most divergent animal mitogenome characterized to date [23], are only $28 \%$ and $36 \%$ identical, respectively, to those of the slowly evolving sponge Xestospongia muta. In contrast, the Viscum album Cob and Cox1 proteins are 69\% and $84 \%$ identical to homologs from the slowly evolving angiosperm Liriodendron tulipifera. This broader perspective supports the hypothesis that, apart from the undisputed loss of all complex I genes, the mitogenomes of $V$. album and other Viscum species possess the same basic set of functional respiratory and ATP-synthesis genes found in all other angiosperms examined to date.

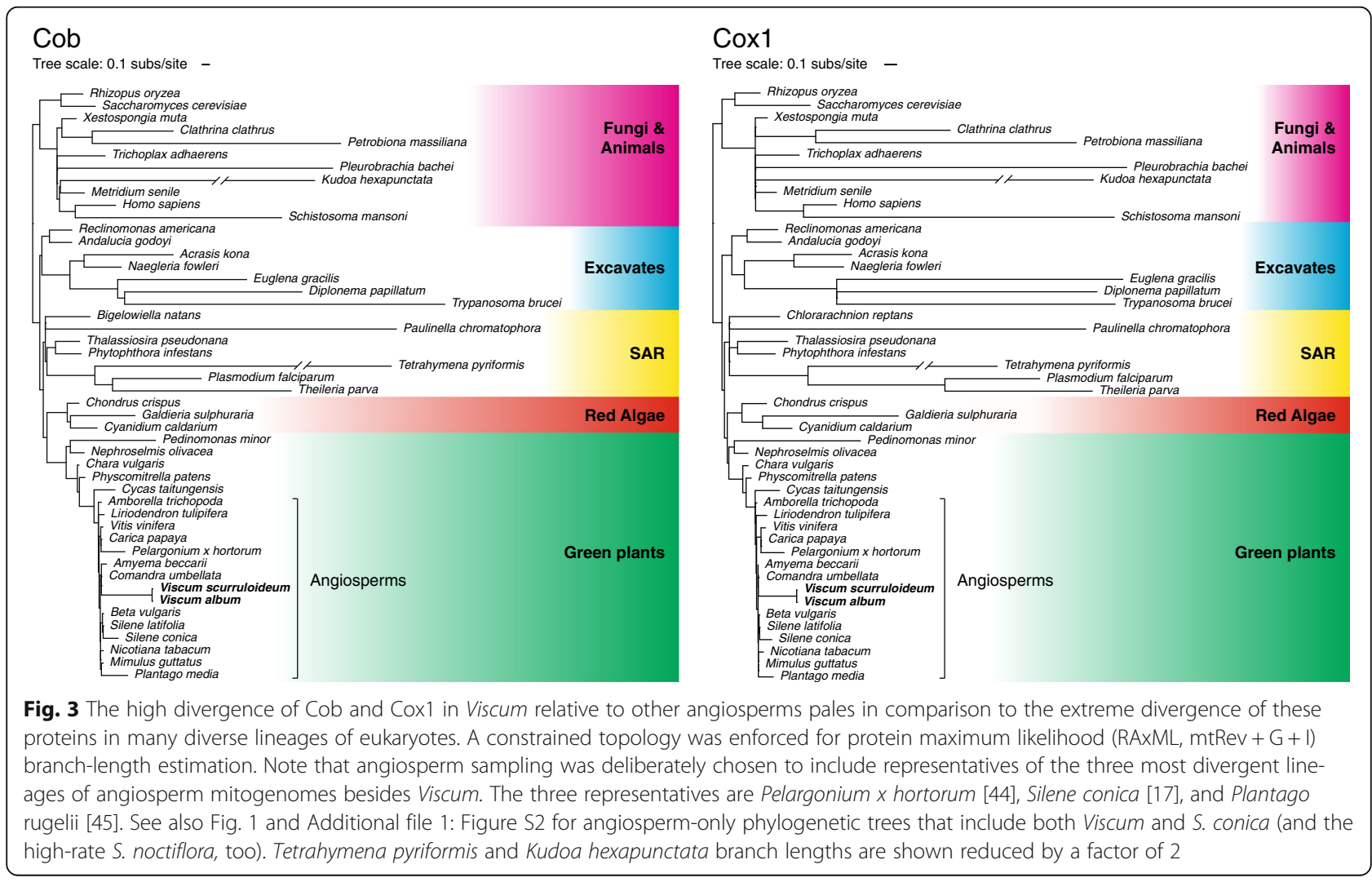




\section{Horizontal gene transfer of $\mathrm{ccmB}$ and matR}

In contrast to the extremely divergent gene sequences typically found in Viscum (Fig. 1 and Additional file 1: Figure S2), the $V$. album ccmB and matR sequences are, as noted by Petersen et al. [5], unexceptional in their level of sequence divergence (Fig. 4). This observation, together with the absence of $c \mathrm{cmB}$ and matR in $V$. scurruloideum [2] and their apparent absence in $V$. minimum and $V$. crassulae [5], suggests that these genes were reacquired recently, on the branch leading to $V$. album. To verify that these two gene sequences belong to $V$. album rather than being the result of contamination, we used PCR to amplify them from an independent sample of $V$. album DNA. Sequencing of these PCR products recovered identical $c \mathrm{cmB}$ and matR sequences to those reported in the $V$. album paper [5], thereby verifying the original report.

\section{matR}

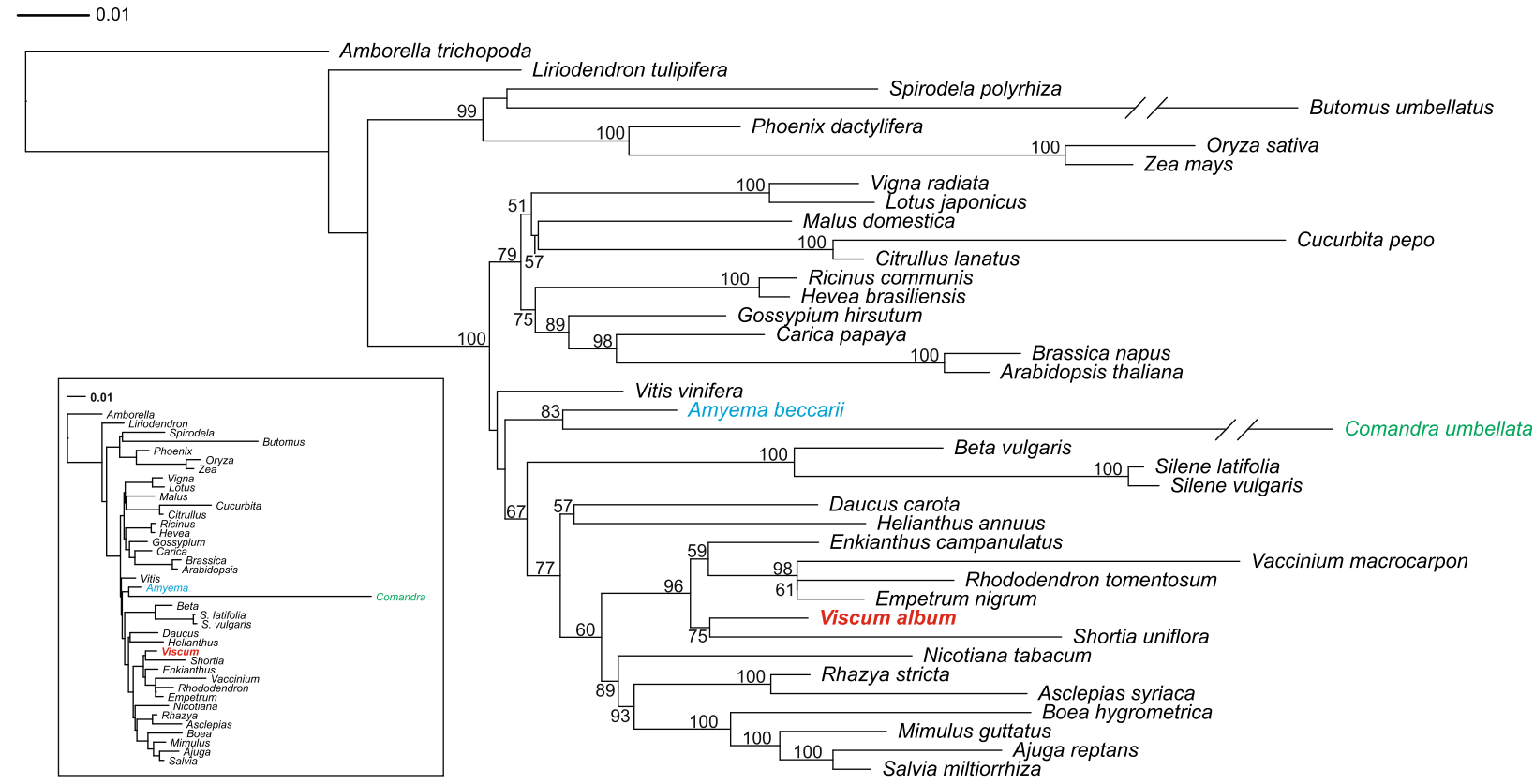

$\mathrm{ccmB}$

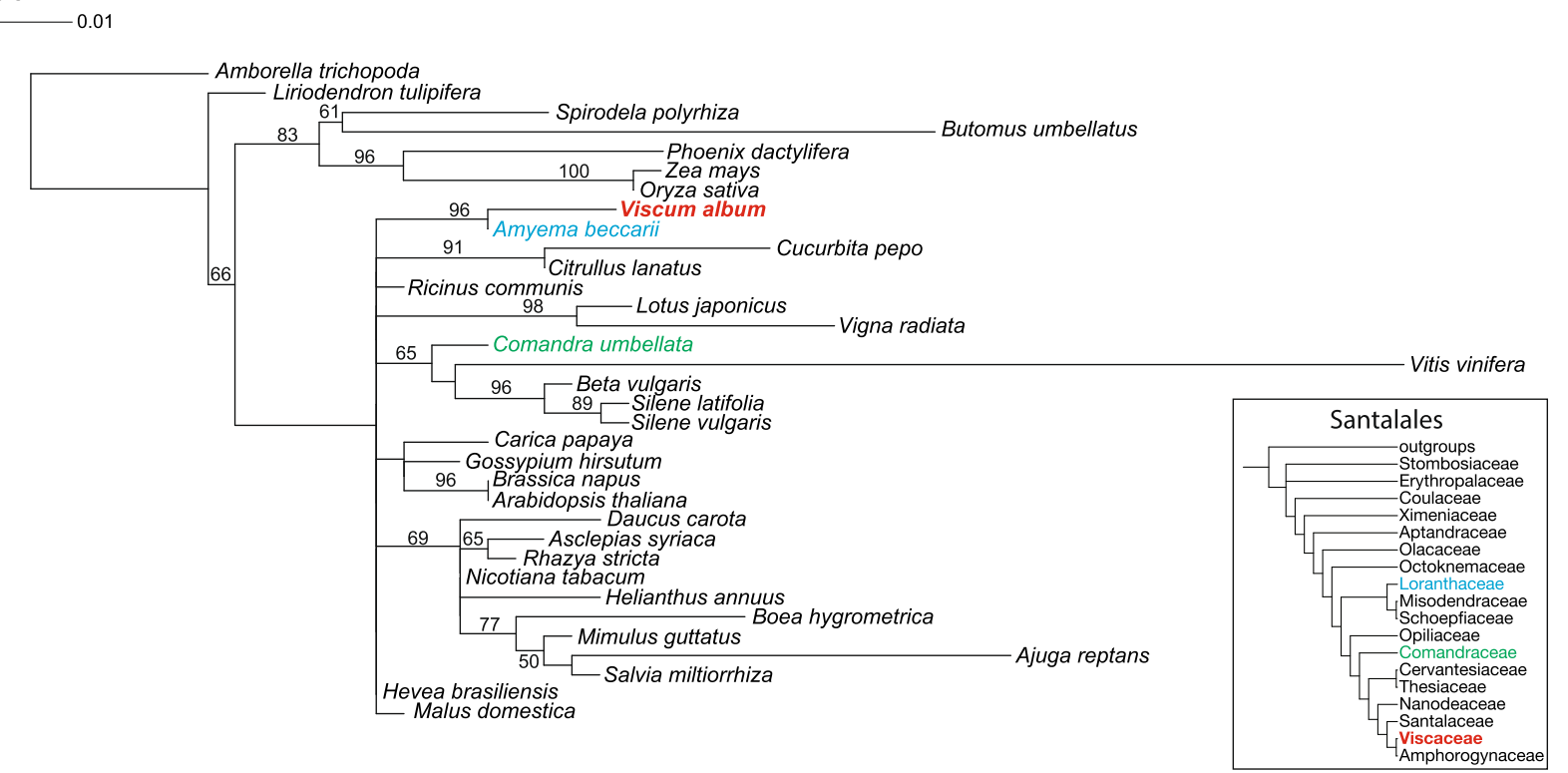

Fig. 4 Evidence for horizontal origin of the matR and $c \mathrm{cmB}$ genes in V. album based on maximum-likelihood analysis of all-position nucleotide alignments. Note that $V$. scurruloideum lacks both of these genes. Bootstrap values $>50 \%$ are shown. The Santalales phylogeny shown inset in the $\mathrm{ccmB}$ tree is the current best estimate of relationships among these plants $[6,7]$ 
Consistent with the above observations, phylogenetic analyses indicate that $V$. album acquired matR and $c c m B$ via HGT, probably from two different donors (Fig. 4). The matR tree placed $V$. album within Ericales with strong support (96\% bootstrap). This order contains, among others, blueberries (Vaccinium), primroses (Primula), and tea (Camellia) (Fig. 4). To assess confidence in the aberrant matR topology shown in Fig. 4, we used the approximately unbiased (AU) test [24] to compare it to an alternative topology, of strictly vertical matR transmission, in which $V$. album matR was constrained to be sister to Comandra matR (see inset cladogram in Fig. 4). This alternative tree was rejected with a $P$ value of 1e-06. These phylogenetic results, in conjunction with the divergence and distributional data, strongly indicate that $V$. album acquired matR recently by HGT, most likely from a member of the Ericales. Viscum album, which is naturally widespread in Europe and central and western Asia, is known to parasitize no fewer than 452 species from 44 families [25]. Consistent with the phylogenetic results, $V$. album has been found to parasitize introduced members of two Ericales families (Ericaceae and Ebenaceae). Although these Ericales species do not occur within the current native range of $V$. album [25], ancestral range overlaps of a parasite and its hosts are difficult to infer in general, and all the more so given that $V$. album currently parasitizes so many diverse hosts.

The $c c m B$ tree placed $V$. album as sister to another member of Santalales, Amyema, also with strong support (96\% bootstrap). This placement is not congruent with Santalales phylogeny [6]; in the context of our sampling, Viscum should instead appear as sister to Comandra (Fig. 4). An alternative topology of strictly vertical transmission of $c c m B$, i.e., in which $V$. album $c c m B$ was constrained as sister to Comandra $c c m B$, was rejected with a $P$ value of $4 \mathrm{e}-4$. These phylogenetic results, taken together with the divergence and distributional findings, strongly indicate that $V$. album acquired its $c \mathrm{cmB}$ gene by recent HGT from another lineage within Santalales. Viscum album is well known [25] to be hyperparasitic on another European aerial hemiparasite, Loranthus europea, a member of the same family (Loranthaceae) as Amyema. Thus, $V$. album may have acquired $c c m B$ from a host plant, itself a parasite.

The $V$. album study [5] noted that the anomalously low level of sequence divergence exhibited by $\operatorname{cm} B$ and matR "may indicate that they are the only truly functional genes" in the mitogenome. However, the phylogenetic and distributional evidence presented above suggest otherwise: The presence and anomalously low divergence of these two genes in $V$. album are most likely the result of two recent HGT events. Accordingly, the intactness of these genes in $V$. album, rather than being evidence for functionality, is probably simply a reflection of their limited residency in the $V$. album mitogenome, i.e., they have not been present in $V$. album long enough to incur pseudogenizing mutations.

\section{Enormous difference in mitogenome size between $V$. album and $V$. scurruloideum}

At $565 \mathrm{~kb}$, the $V$. album mitogenome [5] is far larger (by a factor of 8.6) than that of its closest sequenced relative, $V$. scurruloideum [2]. Much of this difference is presumably due to genome compaction in $V$. scurruloideum, which at $66 \mathrm{~kb}$ is the smallest (by over 3-fold) angiosperm mitogenome sequenced to date [2], whereas $565 \mathrm{~kb}$ is close to the median size of currently examined angiosperm mitogenomes (Additional file 1: Figure S1). This level of within-genus variation in mitogenome size is unusual for any group of eukaryotes, even angiosperms, long known to sustain massive and enigmatic changes in mitogenome size $[17,26]$. Nine of the 10 other multiply sampled angiosperm genera exhibit less than two-fold variation in mitogenome size (http://www.ncbi.nlm. nih.gov/genome/organelle/), while the Viscum size difference exceeds that of all but one of the seven other angiosperm families for which multiple genera have been sampled. Even more exceptional than Viscum is the genus Silene (Caryophyllaceae), in which mitogenome size varies by a factor of 45 (from $253 \mathrm{~kb}$ to 11.3 Mb) among four examined species [17].

\section{Conclusions}

Viscum mitogenomes are extraordinary compared to those of other angiosperms. Our reannotation and reanalysis of the $V$. album mitogenome has confirmed that the unprecedented loss of respiratory complex I in a multicellular organism is not restricted to V. scurruloideum [2] and probably occurred sometime prior to the divergence of this species and $V$. album (and also the other two Viscum species examined [5]). As well, both Viscum genomes are exceedingly divergent at the sequence level due to a combination of highly elevated synonymous substitution rates and relaxed selection. Viscum scurruloideum possesses other unusual properties that are either not shared by $V$. album (i.e., a highly miniaturized genome) or remain uninvestigated (i.e., unusually high levels of recombination across short repeats and of sublimons) [2].

Although the $V$. album mitogenome is extraordinary in certain ways, we find no evidence that it has sustained unprecedented events of gene loss or disabling divergence involving mitochondrial rRNAs or proteins fundamental to respiratory complexes III and IV and the mitochondrial ATP synthase. Instead, the presence in both Viscum mitogenomes of the same complement of essentially full-length respiratory genes that are all under some level of purifying selection, coupled with full-length rRNA sequences that 
exhibit compensatory mutations and canonical predicted secondary structures, indicates that respiratory and translational functions are almost certainly maintained in the Viscum lineage. Our findings emphasize the critical importance of conducting BLAST homology searches in highly divergent sequences with appropriately sensitive parameter settings in order to achieve accurate annotations.

The results of this study point to several directions for future research. First, the occurrence of multicellular aerobic respiration without complex I points to a need for additional functional analysis of the role of NADH oxidation. Second, the Viscum lineage exhibits a fascinating combination of sequence divergence and repeat-mediated recombination potential that could allow future studies to uncover mechanisms of genome size variation. Finally, the discovery of HGT in the mitogenome of $V$. album - one of the most familiar and common parasitic plants - may enable it to serve as a tractable system in which to study the dynamics of foreign sequence acquisition and integration.

\section{Methods}

\section{Gene annotation}

The two strategies used to detect protein and rRNA genes in the $V$. album mitogenome are described in the first section of Results and Discussion. tRNA genes were predicted using tRNAscan version 1.23 [27].

\section{Phylogenetic analysis and evolutionary rate estimation}

Throughout this paper, Viscum gene divergence is reported with respect to Liriodendron. This is because the extraordinarily low genome-wide silent substitution rate [28] of the Liriodendron mitogenome makes it an ideal reference genome for comparative sequence analyses in angiosperms. All alignments were made using MAFFT v. 7.130b [29] with the L-INS-I option. All protein-gene sets were initially aligned at the amino acid level and then either analyzed as such (for the eukaryote-wide analyses shown in Fig. 2) or computationally converted to nucleotide alignments using PAL2NAL [30] such that the resulting arrangement of nucleotide triplets reflected the corresponding protein alignment. Codons empirically determined to undergo mitochondrial RNA editing in at least one of eight angiosperms (Arabidopsis thaliana [31], Beta vulgaris [32], Brassica napus [33], Citrullus lanatus [34], Cucurbita pepo [34], Oryza sativa [35], Silene latifolia [36], and Vitis vinifera [37]) or predicted (using PREPMitochondrial [38] with a stringency setting of 0.2) to undergo RNA editing in V. scurruloideum, Amyema, or Comandra were excluded from all nucleotide analyses. All phylogenetic trees constructed from nucleotide alignments were estimated with RAxML v. 7.2.8 [39] using the generalized time-reversible (GTR) model with gamma correction for among-site rate variation and 10 starting trees. Support for nodes was assessed using 1,000 bootstrap replicates.
The topologically constrained amino-acid trees shown in Fig. 3 were also estimated with RAxML, but under the mtRev + G + I model.

PAML's codeml [40] was used to estimate $d_{S}$ and $d_{N}$ values as described previously [2]. A simplified GoldmanYang codon model was used with separate branch $d_{N} / d_{S}$ ratios $(\omega)$ that allowed for the following seven sets of branches: the $V$. scurruloideum branch, the $V$. album branch; the $V$. minimum branch, the $V$. crassulae branch; the ancestral Viscum branch; the S. conica and S. noctiflora branches (one set); and all remaining branches.

To test for differences in selection pressure between Viscum and non-Viscum branches of protein-gene trees, we used a codon-based model-testing framework implemented in RELAX [22], available on Datamonkey [41].

\section{Alternative topology tests}

The CONSEL package [42] was used to calculate the approximately unbiased (AU) $P$ values for unconstrained and constrained trees. To generate constrained ML trees for matR and $c c m B$, we required $V$. album to be sister to Comandra, and conducted ML searches under this constraint using RAxML v. 7.2.8 [42].

\section{PCR and DNA sequencing}

Total DNA from $V$. album was extracted using the DNeasy kit (Qiagen) from $20 \mathrm{mg}$ of leaf tissue collected from a plant growing on an apple tree in Oviedo, Spain. We performed PCR to amplify matR and $c \mathrm{cmB}$ from $V$. album using the following primers: $m a t R$-forward, GTTTTCACACCATC GACCGACATCG; matR-reverse, CGCGGCACCTGTAG TAGGACAGAGGA; $\quad c c m B$-forward, CATGTCATTCCCA TTTAGGTCCG; and $c c m B$-reverse: GGTGAAGTGGTTG GATTTAGCG. Thermal cycling conditions included an initial hold at $94{ }^{\circ} \mathrm{C}$ for $2^{\prime} 30^{\prime \prime}$ followed by 35 cycles of $94{ }^{\circ} \mathrm{C}$ for $30^{\prime \prime}, 50^{\circ} \mathrm{C}$ for $1^{\prime}$, and $72^{\circ} \mathrm{C}$ for $1^{\prime} 30^{\prime \prime}$. Amplification products were purified using the QIAquick PCR Purification kit (Qiagen), and both strands were sequenced.

\section{Additional file}

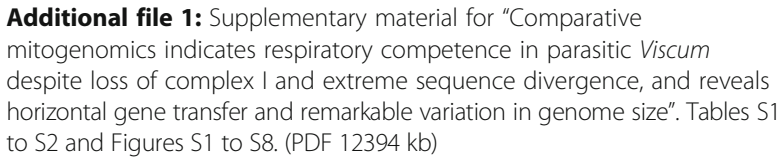

\section{Abbreviations}

AU: Approximately unbiased; GTR: Generalized time-reversible; HGT: Horizontal gene transfer; HSPs: High-scoring segment pairs; LSU: Large subunit; SSU: Small subunit

\section{Acknowledgements}

We thank Marcos Caraballo, Tomas Carlo, and Claude dePamphilis (all at Pennsylvania State University) for providing the sample of $V$. album DNA, and Dennis Lavrov (at lowa State University) for the Petrobiona massiliana cob and cox 1 sequences. 


\section{Funding}

This work was supported by National Science Foundation award IOS-1027529 to J.D.P.

\section{Availability of data and materials}

The $V$. album and $V$. scurruloideum mitogenome sequences are available from the Genbank database, www.ncbi.nlm.nih.gov/genbank/ (accession nos. NC029039, KT022222 and KT022223).

\section{Author contributions}

ES, TJB, DWR and JDP designed research; ES performed research; ES, TJB, DWR, and JDP analyzed data; and ES and JDP wrote the paper. All authors read and approved the final manuscript.

\section{Competing interests}

The authors declare that they have no competing interests.

\section{Consent for publication}

Not applicable.

\section{Ethics approval and consent to participate}

Not applicable.

\section{Author details}

${ }^{1}$ Department of Biology, Indiana University, Bloomington, IN 47405, USA. ${ }^{2}$ Department of Biological Sciences, Western Michigan University, Kalamazoo, MI 49008, USA. ${ }^{3}$ Present address: Department of Bioinformatics and Computational Biology, Genentech Inc., 1 DNA Way, South San Francisco, CA 94080, USA.

Received: 6 September 2016 Accepted: 31 January 2017 Published online: 21 February 2017

\section{References}

1. Flegontov P, Michalek J, Janouskovec J, Lai DH, Jirků M, Hajdušková E, et al. Divergent mitochondrial respiratory chains in phototrophic relatives of apicomplexan parasites. Mol Biol Evol. 2015;32:1115-31.

2. Skippington E, Barkman TJ, Rice DW, Palmer JD. Miniaturized mitogenome of the parasitic plant Viscum scurruloideum is extremely divergent and dynamic and has lost all nad genes. Proc Natl Acad Sci U S A. 2015;112:E3515-24.

3. Adams KL, Qiu YL, Stoutemyer M, Palmer JD. Punctuated evolution of mitochondrial gene content: high and variable rates of mitochondrial gene loss and transfer to the nucleus during angiosperm evolution. Proc Natl Acad Sci U S A. 2002:99:9905-12.

4. Mower JP, Sloan DB. Alverson AJ Plant mitochondrial diversity-the genomics revolution. In: Wendel JF, Greilhuber J, Dolezel I, Leitch IJ, editors. Plant Genome Diversity. Berlin: Springer; 2014. p. 123-44.

5. Petersen G, Cuenca A, Moller IM, Seberg O. Massive gene loss in mistletoe (Viscum, Viscaceae) mitochondria. Sci Rep. 2015;5:17588.

6. Der JP, Nickrent DL. A molecular phylogeny of Santalaceae (Santalales). Syst Bot. 2008;33:107-16.

7. Su HJ, Hu JM, Anderson FE, Der JP, Nickrent DL. Phylogenetic relationships of Santalales with insights into the origins of holoparasitic Balanophoraceae. Taxon. 2015;64:491-506.

8. Camacho C, Coulouris G, Avagyan V, Ma N, Papadopoulos J, Bealer K, et al. BLAST+: architecture and applications. BMC Bioinformatics. 2009;10:421.

9. Copley RR, Goodstadt L, Ponting C. Eukaryotic domain evolution inferred from genome comparisons. Curr Opin Genet Dev. 2003;13:623-8.

10. Rost B. Twilight zone of protein sequence alignments. Protein Eng. 1999;12:85-94.

11. Adams KL, Palmer JD. Evolution of mitochondrial gene content: gene loss and transfer to the nucleus. Mol Phylogenet Evol. 2003;29:380-95.

12. Klimov PB, Knowles LL. Repeated parallel evolution of minimal rRNAs revealed from detailed comparative analysis. J Hered. 2011;102:283-93.

13. Boer PH, Gray MW. Scrambled ribosomal RNA gene pieces in Chlamydomonas reinhardtii mitochondrial DNA. Cell. 1988;55:399-411.

14. Feagin JE, Harrell MI, Lee JC, Coe KJ, Sands BH, Cannone JJ, et al. The fragmented mitochondrial ribosomal RNAs of Plasmodium falciparum. PLoS One. 2012;7, e38320.

15. Gutell RR, Weiser B, Woese CR, Noller HF. Comparative anatomy of 16S-like ribosomal RNA. Prog Nucleic Acid Res Mol Biol. 1985;32:155-216.
16. Valach M, Burger G, Gray MW, Lang BF. Widespread occurrence of organelle genome-encoded $5 \mathrm{~S}$ rRNAs including permuted molecules. Nucleic Acids Res. 2014;42:13764-77.

17. Sloan DB, Alverson AJ, Chuckalovcak JP, Wu M, McCauley DE, Palmer JD, et al. Rapid evolution of enormous, multichromosomal genomes in flowering plant mitochondria with exceptionally high mutation rates. PLoS Biol. 2012;10, e1001241.

18. Hancock K, Hajduk SL. The mitochondrial tRNAs of Trypanosoma brucei are nuclear encoded. J Biol Chem. 1990:265:19208-15.

19. Shi $X$, Chen $\mathrm{DH}$, Suyama $\mathrm{Y}$. A nuclear tRNA gene cluster in the protozoan Leishmania tarentolae and differential distribution of nuclear-encoded tRNAs between the cytosol and mitochondria. Mol Biochem Parasitol. 1994;65:23-37.

20. Sharma A, Sharma A. Plasmodium falciparum mitochondria import tRNAs along with an active phenylalanyl-tRNA synthetase. Biochem J. 2015;465: 459-69.

21. Schmidt O, Pfanner N, Meisinger C. Mitochondrial protein import: from proteomics to functional mechanisms. Nat Rev Mol Cell Biol. 2010;11:655-67.

22. Wertheim JO, Murrell B, Smith MD, Kosakovsky Pond SL, Scheffler K. RELAX: detecting relaxed selection in a phylogenetic framework. Mol Biol Evol. 2015;32:820-32.

23. Takeuchi F, Sekizuka T, Ogasawara Y, Yokoyama H, Kamikawa R, Inagaki Y, et al. The mitochondrial genomes of a Myxozoan genus Kudoa are extremely divergent in Metazoa. PLoS One. 2015;10, e0132030.

24. Shimodaira H. An approximately unbiased test of phylogenetic tree selection. Syst Biol. 2002;51:492-508.

25. Barney CW, Hawksworth FG, Geils BS. Hosts of Viscum album. Forest Pathol. 1998;28:187-208.

26. Ward BL, Anderson RS, Bendich AJ. The mitochondrial genome is large and variable in a family of plants (Cucurbitaceae). Cell. 1981;25:793-803.

27. Lowe TM, Eddy SR. tRNAscan-SE: a program for improved detection of transfer RNA genes in genomic sequence. Nucleic Acids Res. 1997;25:955-64.

28. Richardson AO, Rice DW, Young GJ, Alverson AJ, Palmer JD. The "fossilized" mitochondrial genome of Liriodendron tulipifera: ancestral gene content and order, ancestral editing sites, and extraordinarily low mutation rate. BMC Biol. 2013;11:29.

29. Katoh K, Standley DM. MAFFT multiple sequence alignment software version 7: improvements in performance and usability. Mol Biol Evol. 2013;30:772-80.

30. Suyama M, Torrents D, Bork P. PAL2NAL: robust conversion of protein sequence alignments into the corresponding codon alignments. Nucleic Acids Res. 2006:34:W609-12.

31. Giege P, Brennicke A. RNA editing in Arabidopsis mitochondria effects $441 \mathrm{C}$ to U changes in ORFs. Proc Natl Acad Sci U S A. 1999;96:15324-9.

32. Mower JP, Palmer JD. Patterns of partial RNA editing in mitochondrial genes of Beta vulgaris. Mol Gen Genomics. 2006;276:285-93.

33. Handa $\mathrm{H}$. The complete nucleotide sequence and RNA editing content of the mitochondrial genome of rapeseed (Brassica napus L.): comparative analysis of the mitochondrial genomes of rapeseed and Arabidopsis thaliana. Nucleic Acids Res. 2003;31:5907-16.

34. Alverson AJ, Wei $X$, Rice DW, Stern DB, Barry K, Palmer JD. Insights into the evolution of mitochondrial genome size from complete sequences of Citrullus lanatus and Cucurbita pepo (Cucurbitaceae). Mol Biol Evol. 2010;27:1436-48.

35. Notsu Y, Masood S, Nishikawa T, Kubo N, Akiduki G, Nakazono M, et al. The complete sequence of the rice (Oryza sativa L.) mitochondrial genome: frequent DNA sequence acquisition and loss during the evolution of flowering plants. Mol Genet Genomics. 2002;268:434-45.

36. Sloan DB, MacQueen AH, Alverson AJ, Palmer JD. Taylor DR. Extensive loss of RNA editing sites in rapidly evolving Silene mitochondrial genomes: selection vs retroprocessing as the driving force Genetics. 2010;185:1369-80.

37. Picardi E, Horner DS, Chiara M, Schiavon R, Valle G, Pesole G. Large-scale detection and analysis of RNA editing in grape mtDNA by RNA deepsequencing. Nucleic Acids Res. 2010;38:4755-67.

38. Mower JP. The PREP suite: predictive RNA editors for plant mitochondrial genes, chloroplast genes and user-defined alignments. Nucleic Acids Res. 2009:37:W253-9.

39. Stamatakis A. RAxML-VI-HPC: maximum likelihood-based phylogenetic analyses with thousands of taxa and mixed models. Bioinformatics. 2006:22:2688-90.

40. Yang Z. PAML 4: Phylogenetic analysis by maximum likelihood. Mol Biol Evol. 2007:24:1586-91.

41. Delport W, Poon AF, Frost SD, Kosakovsky Pond SL. Datamonkey 2010: a suite of phylogenetic analysis tools for evolutionary biology. Bioinformatics. 2010:26:2455-7. 
42. Shimodaira H, Hasegawa M. CONSEL: for assessing the confidence of phylogenetic tree selection. Bioinformatics. 2001;17:1246-7.

43. Alikhan NF, Petty NK, Ben Zakour NL, Beatson SA. BLAST Ring Image Generator (BRIG): simple prokaryote genome comparisons. BMC Genomics. 2011;12:402.

44. Parkinson CL, Mower JP, Qiu YL, Shirk AJ, Song K, Young ND, et al. Multiple major increases and decreases in mitochondrial substitution rates in the plant family Geraniaceae. BMC Evol Biol. 2005;5:73.

45. Cho Y, Mower JP, Qiu YL, Palmer JD. Mitochondrial substitution rates are extraordinarily elevated and variable in a genus of flowering plants. Proc Natl Acad Sci U S A. 2004;101:17741-6.

Submit your next manuscript to BioMed Central and we will help you at every step:

- We accept pre-submission inquiries

- Our selector tool helps you to find the most relevant journal

- We provide round the clock customer support

- Convenient online submission

- Thorough peer review

- Inclusion in PubMed and all major indexing services

- Maximum visibility for your research

Submit your manuscript at www.biomedcentral.com/submit
Biomed Central 